\title{
Structuring Families of Industrial Case Studies
}

\author{
Laurie Williams
}

Practitioners are most influenced by results of research conducted in industrial settings. Evidence of the efficacy of a software development practice or process is best obtained through a triangulation of research findings obtained through a variety of empirical studies in various contexts. The use of an evaluation framework can enable a family of related industrial case studies in different contexts to be metaanalyzed and/or combined. Such an evaluation framework could consists of templates for specific quantitative measures to collect with associated instructions on what to include/exclude for consistent measurement collection as well as protocols for surveys and/or interviews. Groups of researchers interested in the same research question(s) can customize and evolve an evaluation framework for the technology under study.

We have developed and evolved such a framework for the study of Extreme Programming, the Extreme Programming Evaluation Framework (XP-EF), which is available as a North Carolina State University Technical Report. XP-EF is currently at Version 1.4. The composition of Version 1.0 was initially developed from relevant literature. We refined the framework in several research cycles with industrial partners on four multi-release industrial case studies. We have also revised the process based upon input from anonymous reviews of our publications from researchers in the community, through discussions with members of the International Software Engineering Research Network (ISERN), and through presentation of our work to researchers and practitioners. Through these cycles of use and external feedback, the research and practitioner communities have provided input on the guidelines and artifacts embodied in the framework. We have conducted four case studies guided by the XP-EF. The reported results of these case studies reference the evaluation framework used, enabling precise replication of metrics collection and data analysis. 